\title{
PROGNOSTIC VALUE OF DIASTOLIC FUNCTION PARAMETERS IN SIGNIFICANT AORTIC REGURGITATION. THE ROLE OF THE LEFT ATRIAL STRAIN.
}

Ana Garcia-Martin ${ }^{1}$, María Abellás², Ariana González Gómez ${ }^{3}$, Luis Rincon Diaz ${ }^{4}$, Juan Manuel Monteagudo-Ruiz ${ }^{2}$, Rocio Hinojar-Baydes ${ }^{5}$, Jose Luis Moya Mur ${ }^{6}$, Jose Zamorano ${ }^{5}$, and Covadonga Fernandez-Golfin ${ }^{5}$

${ }^{1}$ Affiliation not available

${ }^{2}$ Hospital Universitario Ramon y Cajal

${ }^{3}$ University Hospital Ramón y Cajal

${ }^{4}$ Hospital Clinico de Salamanca

${ }^{5}$ Ramon y Cajal University Hospital

${ }^{6}$ Hospital Universitario Ramón y Cajal

September 25, 2021

\begin{abstract}
Aims. The management of patients with asymptomatic significant aortic regurgitation (sAR) is often challenging and appropriate timing of aortic valve surgery remains controversial. Prognostic value of diastolic parameters has been demonstrated in several cardiac diseases. In particular, left atrial (LA) function has been shown to be an important determinant of morbimortality. The purpose of this study was to analyze the prognostic significance of diastolic function in asymptomatic patients with sAR. Methods and results. A total of 126 patients with asymptomatic sAR were included. Conventional echocardiographic systolic and diastolic function parameters were assessed. LA auto-strain analysis was performed in a subgroup of 57 patients. During a mean follow up of $33 \pm 19$ months, 25 (19,8\%) patients reached the combined end-point. Univariate analysis showed that LV volumes, LVEF, E wave, E/e' ratio, LA volume and LA reservoir strain (LASr) were significant predictors of events. Multivariate model 1 that tested all echocardiographic variables statistically significant in the univariate model showed that LVEDV [HR=1,02;95\% CI:1,01-1,03 ( $\mathrm{p}<0,001)]$ and E/e' ratio $[\mathrm{HR}=1,12 ; 95 \%$ CI:1,03-1,23 ( $\mathrm{p}=0,01)]$ were significant predictors of events. In the subgroup of patients with LA auto-strain analyzed, a second multivariate model including the previous significant variables for the first model as well as LASr, showed that LVEDV [HR=1,05;95\% CI:1,01-1,08 ( $\mathrm{p}=0,016)]$ and LASr $[\mathrm{HR}=0,8 ; 95 \% \mathrm{CI}: 0,65-0,98(\mathrm{p}<0,035)]$ were the most significant predictors of cardiovascular events. Conclusions. In this population of asymptomatic patients with sAR and normal LV systolic function, baseline diastolic parameters were prognostic markers of cardiovascular events; among them, LASr played a strong independent predictor role.
\end{abstract}

\section{PROGNOSTIC VALUE OF DIASTOLIC FUNCTION PARAMETERS IN SIGNIFICANT AORTIC REGURGITATION. THE ROLE OF THE LEFT ATRIAL STRAIN.}

Ana García Martín MD, $\mathrm{PhD}^{1}$, María Abellás Sequeiros $\mathrm{MD}^{1}$, Ariana González Gómez $\mathrm{MD}^{1}$, Luis Miguel Rincón Díaz MD², Juan Manuel Monteagudo Ruiz MD ${ }^{1}$, Rocío Hinojar Baydés MD, $\mathrm{PhD}^{1}$, Jose Luis Moya Mur MD, $\mathrm{PhD}^{1}$, José Luis Zamorano Gómez MD, $\mathrm{PhD}^{1}$, Covadonga Fernández-Golfín MD, $\mathrm{PhD}^{1}$.

${ }^{1}$ Department of Cardiology. Ramón y Cajal University Hospital. Madrid. Spain. CIBER CV. 
${ }^{2}$ Department of Cardiology. University Hospital. Salamanca. Spain.

Address:

Ana García Martín. Department of Cardiology. Ramón y Cajal University Hospital. Ctra. Colmenar Km 9,100. 28034 Madrid, Spain. Telephone number: +34913368259. E-mail:aggarciamartin@gmail.com

María Abellás Sequeiros. Department of Cardiology. Ramón y Cajal University Hospital. Ctra. Colmenar Km 9,100. 28034 Madrid, Spain. Telephone number: +34913368259. E-mail: maine.iaca@gmail.com

Ariana González Gómez. Department of Cardiology. Ramón y Cajal University Hospital. Ctra. Colmenar Km 9,100. 28034 Madrid, Spain. Telephone number: +34913368259. E-mail: arianaglz@hotmail.com

Luis Miguel Rincón Díaz. Department of Cardiology. University Hospital. Salamanca. Spain. Email:lmrincondiaz@hotmail.com

Juan Manuel Monteagudo Ruiz. Department of Cardiology. Ramón y Cajal University Hospital. Ctra. Colmenar Km 9,100. 28034 Madrid, Spain. Telephone number: +34913368259. E-mail: j5469m@gmail.com

Rocío Hinojar Baydés. Department of Cardiology. Ramón y Cajal University Hospital. Ctra. Colmenar Km 9,100. 28034 Madrid, Spain. Telephone number: +34913368259. E-mail: rociohinojar@gmail.com

José-Luis Moya-Mur. Department of Cardiology. Ramón y Cajal University Hospital. Ctra. Colmenar Km 9,100. 28034 Madrid, Spain. Telephone number: +34913368259. E-mail: joseluis.moya@salud.madrid.org

Covadonga Fernández-Golfín. Department of Cardiology. Ramón y Cajal University Hospital. Ctra. Colmenar Km 9,100. 28034 Madrid, Spain. Telephone number: +34913368259. E-mail: covagolfin@yahoo.es

José Luis Zamorano-Gómez. Department of Cardiology. Ramón y Cajal University Hospital. Ctra. Colmenar Km 9,100. 28034 Madrid, Spain. Telephone number: +34913368259. E-mail: zamorano@secardiologia.es

\section{ABSTRACT.}

Aims.

The management of patients with asymptomatic significant aortic regurgitation (sAR) is often challenging and appropriate timing of aortic valve surgery remains controversial. Prognostic value of diastolic parameters has been demonstrated in several cardiac diseases. In particular, left atrial (LA) function has been shown to be an important determinant of morbimortality. The purpose of this study was to analyze the prognostic significance of diastolic function in asymptomatic patients with sAR.

\section{Methods and results.}

A total of 126 patients with asymptomatic sAR were included. Conventional echocardiographic systolic and diastolic function parameters were assessed. LA auto-strain analysis was performed in a subgroup of 57 patients. During a mean follow up of $33 \pm 19$ months, $25(19,8 \%)$ patients reached the combined end-point. Univariate analysis showed that LV volumes, LVEF, E wave, E/e' ratio, LA volume and LA reservoir strain (LASr) were significant predictors of events. Multivariate model 1 that tested all echocardiographic variables statistically significant in the univariate model showed that LVEDV [HR $=1,02 ; 95 \%$ CI:1,01-1,03 $(\mathrm{p}<0,001)]$ and $\mathrm{E} / \mathrm{e}$ ' ratio $[\mathrm{HR}=1,12 ; 95 \% \mathrm{CI}: 1,03-1,23(\mathrm{p}=0,01)]$ were significant predictors of events. In the subgroup of patients with LA auto-strain analyzed, a second multivariate model including the previous significant variables for the first model as well as LASr, showed that LVEDV [HR=1,05;95\% CI:1,01-1,08 ( $\mathrm{p}=0,016)]$ and LASr $[\mathrm{HR}=0,8 ; 95 \%$ CI:0,65-0,98 $(\mathrm{p}<0,035)]$ were the most significant predictors of cardiovascular events.

\section{Conclusions.}

In this population of asymptomatic patients with sAR and normal LV systolic function, baseline diastolic parameters were prognostic markers of cardiovascular events; among them, LASr played a strong independent predictor role. 


\section{KEYWORDS.}

Echocardiography, aortic regurgitation, diastolic function, strain.

\section{ABBREVIATIONS.}

AUC: Area under the curve.

\section{LA: Left Atrial.}

LASct: Left atrial strain contractile.

LASr: Left atrial strain reservoir.

LAScd: Left atrial strain conduit.

LV: Left ventricle.

LVEF: Left ventricle ejection fraction.

sAR: Significant aortic regurgitation.

SPAP: Systolic pulmonary artery pressure.

TTE: Transtoracic echocardiography.

TR: Tricuspid regurgitation.

\section{INTRODUCTION.}

The management of patients with asymptomatic significant aortic regurgitation (sAR) is often challenging and appropriate timing of aortic valve surgery remains controversial.

According to the latest guidelines, among patients with sAR, surgery is formally recommended when it causes symptoms, left ventricular ejection fraction (LVEF) [?]50\% or if there is a concomitant indication for cardiac surgery (i.e. significant enlargement of ascending aorta). In addition, surgery should be considered in asymptomatic patients with severe LV dilation (end-diastolic diameter $>70 \mathrm{~mm}$ or end-systolic $>50 \mathrm{~mm}$ ) (1).

There are no strong indicators to recommend early surgery in patients with sAR, however delaying the time for the intervention could bring potential negative consequences, such as the risk of permanent LV dysfunction, heart failure or sudden cardiac death (2). In this context, the identification of markers that are early altered in the natural history of the sAR, could be useful in order to improve the management of these asymptomatic patients.

The prognostic value of diastolic parameters has been demonstrated in several cardiac diseases. In particular, left atrial (LA) function has been shown to be an important determinant of morbimortality. More specifically, LA strain is a promising tool for the detection of subclinical cardiac dysfunction (3).

The purpose of this study was to analyze the prognostic significance of diastolic function parameters, included LA strain, in asymptomatic patients with sAR and to evaluate whether these parameters could help to identify patients at high risk of adverse events that could benefit from early cardiac surgery.

\section{METHODS.}

From February 2013 to November 2019 consecutive asymptomatic patients with chronic sAR evaluated in the Heart Valve Clinic with a comprehensive transthoracic echocardiogram (TTE) were included.

Exclusion criteria included patients who already met criteria for cardiac surgery at the time of the inclusion (presence of symptoms, LV dilation or dysfunction, indication for other cardiac surgery), other concomitant significant valve disease (more than mild degree evaluated with echocardiography), LV dysfunction, the presence of atrial fibrillation or pacemaker rhythm. 
Local Ethical Committee approved the study.

\section{Echocardiogram protocol analysis.}

The closest TTE study to the first medical visit in the Heart Valve Clinic was used as baseline analysis. TTE was performed using the commercially available echo systems in the Imaging Unit, according to the standard protocol $(7,8)$. Measurements were reviewed and reperformed by trained echocardiographers.

The severity of AR was assessed using an integrated method including a combination of several measurements (vena contracta width, pressure half-time of the AR jet, the presence of diastolic retrograde flow in aorta, and the LV size) according to the established practice guidelines (9). Aortic regurgitation was considered significant by the presence of the following criteria: the width of the vena contracta $>6 \mathrm{~mm}$, pressure half-time $<200 \mathrm{~ms}$, the presence of diastolic retrograde flow in aorta and some degree of LV enlargement.

LV size was evaluated by its diameters from the parasternal long axis view and by its volumes from the apical four chamber view. LVEF was measured using Teicholz method and apical biplane Simpson's method.

LV diastolic function was evaluated following the established recommendations from the American Society of Echocardiography and the European Association of Cardiovascular Imaging (10), that included the following parameters:

Transmitral flow was recorded by pulsed wave Doppler echocardiography, from apical four chamber view, by placing the sample volume at the tips of the mitral leaflets. Peak E wave velocity, peak A wave velocity, $\mathrm{E}$ to $\mathrm{A}$ wave ratio $(\mathrm{E} / \mathrm{A})$ and $\mathrm{E}$ wave deceleration time were determined.

Tissue Doppler imaging was performed in the apical four chamber view and pulsed wave Doppler sample volume was place in the septal and lateral mitral annulus. The peak annular systolic wave velocities were recorded (e' septal and e' lateral). From these measurements, the mitral E/e' septal and E/e' lateral ratios were calculated, as well as the mean of both results (E/e' ratio).

The severity of tricuspid regurgitation (TR) was assessed using an integrated method, according to the established practice guidelines (9). The systolic pulmonary artery pressure (SPAP) was estimated using TR peak velocity and right atrial pressure, which was estimated by the inferior vena cava diameter from long-axis subxiphoid view and its response to inspiration.

LA size was measured at its antero-posterior diameter from the long axis parasternal view in the end-systolic period, and by LA volume, that was measured using the biplane area length method.

In a subgroup of patients with TTE performed in the Philips stations, LA auto-strain analysis was performed with an offline workstation (autostrain TOMTEC). From the apical four chamber view focus in the LA with a minimum loop length of 2 beats; the software uses Advanced Automatic View Recognition technology to identify LA and automatic contour placement, which automatically detects and places the LA border. The software automatically provides the measurement of the average strain for the three major LA function throughout the cardiac cycle: reservoir (LASr), conduit (LAScd) and contractile (LASct) values. The reference point for deformation analysis was at end diastole (Figure 1).

\section{Clinical outcomes.}

Combined clinical endpoint included hospital admission due to heart failure, cardiovascular mortality, or aortic valve surgery due to have reached the indications for surgery and excluding those undergoing surgery due to aortic dilatation or dissection. Clinical data was obtained from hospital's records.

\section{STATISTICAL ANALYSIS.}

Continuous variables were expressed as mean + - SD. Categoric variables were described as number and percentage. For comparison 2 normally distributed variables, Student $t$ test for continuous variables and chi-squared test for categorical variables were used. The analysis was adjusted for age and sex. 
In order to evaluate diastolic parameters that could serve as early markers of cardiovascular outcome, several statistical analyses were performed. The following Cox regression models were constructed: a) a univariate model with all the variables of interest collected; b) a multivariate model including parameters currently used to indicate surgery (LVEDV, LVESV and LVEF) and the most relevant conventional diastolic function parameters that were statistically associated with the composite endpoint in the univariate analysis (E, $\mathrm{E} / \mathrm{e}$ ' ratio and LA volume; SPAP was excluded in the analysis because its echocardiographic evaluation was only possible in 39 patients). The backward elimination method was used for selection of covariates; c) for the subgroup of patients with TTE performed in a Philips station, a second multivariate model, including the significant variables in the previous model and LA strain, was constructed. LASr was the LA strain parameter used for the analysis for being the strongest LA strain parameter available with more reliable scientific evidence.

Area under the receiver-operating characteristic curve (AUC) was used to evaluate LASr accuracy to predict cardiovascular events. The Kaplan-Meier survival curves for patients above and below the median value of LASr were performed to summarize the follow-up experience of the study population. Interaction was studied. This was a hypothesis-generating and exploratory study; therefore, no formal sample size calculation was performed in advance.

The statistical analyses were performed using SPSS software version 26.0 (SPSS Inc, Chicago, Illinois, United States) and $\mathrm{R}$ version 3.3.2.

\section{RESULTS.}

A total of 126 patients were included. Mean age of the patients was $70+-17,0$ years. Among them, 25 $(19,8 \%)$ had bicuspid valve, $75(59,5 \%)$ were men, 78 (62,4\%) had hypertension, $44(35,3 \%)$ suffered from dyslipidemia and $11(9,6 \%)$ had concomitant coronary artery disease with preserve LVEF.

During a mean follow up of 33+-19 months, 25 (19,8\%) patients reached the combined endpoint $(\mathrm{n}=5$ developed heart failure, $n=25$ underwent surgery, and $n=4$ died due to cardiovascular etiology). Another 3 patients that had undergone valve surgery exclusively due to aortic dilatation and another 7 patients that had died from an extracardiac etiology were not included in the cardiovascular event group. Clinical and echocardiographic characteristics of both groups (control and cardiovascular event group) are displayed in table 1.

Univariate analysis showed that LV volumes, LVEF, E wave, E/e' ratio, LA volume, PSAP and LASr were significant predictors of events, whereas LA diameter and LV diastolic diameter were not. Statistical significance was maintained in the analysis adjusted for age and sex (Table 2). Multivariate model 1 that tested all echocardiographic variables statistically significant in the univariate model showed that the E/e' ratio $(\mathrm{p}=0,01)$ and LVEDV $(\mathrm{p}<0,001)$, were significant predictors of events (Table 2$)$.

In a sub-group of 57 patients LA auto-strain analysis was obtained. During the same follow-up period, $8(14 \%)$ patients developed the combined endpoint (7 patients underwent surgery, one of them developed previous heart failure and died due to cardiovascular etiology). Another 3 patients that underwent valve surgery exclusively due to aortic dilatation were excluded from the cardiovascular event group. In this subgroup of patients with LA autostrain analyzed, a second multivariate model was built, including the previous significant variables for the first model (LVEDV and E/e' ratio) and the LASr. This model showed that LVDEV $(\mathrm{p}=0,016)$ and $\operatorname{LASr}(\mathrm{p}=0,035)$ were the most significant predictors of cardiovascular events (Table 2).

Area under the receiver-operating characteristic (AUC) curve was used to evaluate LASr accuracy to predict cardiovascular events $(\mathrm{AUC}=0,78 \mathrm{p}=0,012)$ (Figure 2). Kaplan Meier curve, stratified by median value of LASr, showed that lower LASr values (less than median of $34 \%$ ) were associated with higher rates of events $(\mathrm{p}=0,013)$ (Figure 3).

\section{DISCUSSION.}


In this population of asymptomatic patients with sAR and normal LV systolic function, baseline diastolic parameters were prognostic markers of cardiovascular events; among them, LASr played a strong independent predictor role. In addition, our results also showed that LV volumes had greater prognostic value that LV diameters in patients with asymptomatic sAR.

In accordance with current clinical practice guidelines (1), aortic valve replacement in patients with sAR should be limited to symptomatic patients or those with LV dysfunction. However, there are possible advantages of an early surgery in valvular heart disease in order to prevent sudden cardiac death, persistent LV systolic dysfunction or worse perioperative and postoperative outcomes. In addition, considering the development of new percutaneous treatments less aggressive than cardiac surgery, identifying those conditions associated with a worse prognosis and who may benefit from an early intervention, seems important.

Previous studies have analyzed the role of the diastolic function in sAR undergoing aortic valve replacement. $M a$ et al described that LV diastolic disfunction, analyzed by the integration of 2 echocardiographic parameters (LA volume index and E/e' ratio) is highly prevalent in patients undergoing aortic valve replacement and might improve after surgery (11). In a similar way, Cayli et al (12) reported that diastolic function is a reliable parameter in predicting outcomes in patients with sAR and LV dysfunction. They found that preoperative diastolic disfunction had an adverse impact on the recovery of the cardiac function after surgery and justify this finding due to the correlation of the myocardial fibrosis and worse diastolic function. Kim et $a l$, also found that preoperative E/e' ratio was correlated with postoperative improvement of LVEF (13).

To the best of our knowledge, this study is the first to evaluate the clinical relevance of baseline diastolic function parameters in patients with asymptomatic sAR. In our sample, E wave, E/e'ratio, SPAP and LA size and function, have significant prognostic value in these patients. Among the analyzed diastolic function parameters, we found that LASr played a strong independent predictor role in outcomes, stronger than LA diameter and LA volume. In patients with sAR, the volume and pressure overload promote LV dilatation and eccentric hypertrophy; in this context, LA function estimated by strain can be reduced even in the absence of LA dilatation, mainly due to interstitial fibrosis, and reflects LV filling pressure. In fact, LA volume has been shown to have low sensitivity in the early detection of LA dysfunction in the setting of LV diastolic dysfunction (14).

There are few data available on AR and LA function and its prognosis implications. Salas Pacheco et al (15) demonstrated that in patients with severe aortic disease (AR and aortic stenosis) LASr was the main variable associated with pulmonary hypertension, they considered that the maintenance of LASr function may be one explanation for the absence of pulmonary hypertension in some patients with severe aortic disease. In isolated aortic stenosis, LA enlargement is recognized as a marker of aortic stenosis severity as well as predictor of postoperative clinical outcomes independently of mean transaortic gradient and LV mass (16). Even more, LASr has been shown as an independent predictor of prognosis in patients with aortic stenosis (17).

We used automated quantification technique for the LA strain assessment, which has the potential advantages of time saving and greater accuracy and reproducibility of the measurements (18). Its implementation could lead to simplify these measurements, making it possible to obtain the deformation parameters in the daily clinical practice.

Finally, although the objective of the study was to analyze diastolic function in patients with sAR, an interesting finding has been that LV volumes have more prognostic value than LV diameters. This finding is in accordance with current guidelines (8) that considered the LV size should be routinely assessed by calculating volumes and the biplane method as the currently recommended 2D method to assess LVEF, however the indications for valve replacement in sAR are still based on LV diameters (1). Previous studies described that LV long axis diameter is closely related with LV systolic and diastolic function in patients with chronic severe AR and that LV long axis function is impaired prior to deterioration of LV global systolic function in these patients, which might indicate subclinical LV dysfunction (19). Therefore, a global assessment of the LV size and function seems necessary, beyond that provided by the determination of 
isolated diameters.

Although further studies, with large sample size, are required to stablish cut off points, we believe that careful assessment of diastolic function including LA strain, could be useful to identify high risk patients who could benefit from shortening follow up periods and early aortic surgery.

\section{LIMITATIONS.}

Our study was an analysis from clinical database, from single referral center with limited number of patients and cardiovascular events. Diastolic parameters are difficult to assess in valvular patients with volume overload; however, they have been analyzed in other volume overload valvulopathies, such as mitral regurgitation, with confirmed prognostic value. Due to the retrospective nature of the study, LA auto-strain was not available throughout the sample. Thus, further prospective studies are required to validate our findings.

\section{CONCLUSION.}

In patients with asymptomatic sAR, baseline diastolic parameters were significant predictors of cardiovascular events in the follow-up. LA reservoir strain was a strong independent prognostic marker. LV volumes had greater prognostic value that $\mathrm{LV}$ diameters in patients with asymptomatic sAR.

Conflict of interest: none declared.

\section{REFERENCES.}

1. Baumgartner H, Falk V, Bax JJ, De Bonis M, Hamm C, Holm PJ, et al; ESC Scientific Document Group. 2017 ESC/EACTS Guidelines for the management of valvular heart disease. Eur Heart J. 2017 Sep 21;38(36):2739-2791.

2. Dujardin KS, Enriquez-Sarano M, Schaff HV, Bailey KR, Seward JB, Tajik AJ. Mortality and morbidity of aortic regurgitation in clinical practice. A long-Term follow-up study. Circulation. 1999 Apr 13;99(14):1851-7.

3. Saraiva RM, Demirkol S, Buakhamsri A, Greenberg N, Popović ZB, Thomas JD, et al. Left atrial strain measured by two-dimensional speckle tracking represents a new tool to evaluate left atrial function. J Am Soc Echocardiogr. 2010 Feb;23(2):172-80.

4. Steadman CD, Ray S, Ng LL, McCann GP. Natriuretic peptides in common valvular heart disease. J Am Coll Cardiol. 2010 May 11;55(19):2034-48.

5. Weber M, Arnold R, Rau M, Elsaesser A, Brandt R, Mitrovic V, et al. Relation of N-terminal pro B-type natriuretic peptide to progression of aortic valve disease. Eur Heart J. 2005 May;26(10):1023-30.

6. Detaint D, Messika-Zeitoun D, Avierinos JF, Scott C, Chen H, Burnett JC Jr, et al. B-type natriuretic peptide in organic mitral regurgitation: determinants and impact on outcome. Circulation. 2005 May 10;111(18):2391-7.

7. Evangelista A, Flachskampf F, Lancellotti P, Badano L, Aguilar R, Monaghan M, et al; European Association of Echocardiography. European Association of Echocardiography recommendations for standardization of performance, digital storage and reporting of echocardiographic studies. Eur J Echocardiogr. 2008 Jul;9(4):438-48.

8. Lang RM, Badano LP, Mor-Avi V, Afilalo J, Armstrong A, Ernande L, et al. Recommendations for cardiac chamber quantification by echocardiography in adults: an update from the American Society of Echocardiography and the European Association of Cardiovascular Imaging. Eur Heart J Cardiovasc Imaging. 2015 Mar;16(3):233- 70.

9. Lancellotti P, Tribouilloy C, Hagendorff A, Popescu BA, Edvardsen T, Pierard LA, et al; Scientific Document Committee of the European Association of Cardiovascular Imaging. Recommendations for the echocardiographic assessment of native valvular regurgitation: an executive summary from the European Association of Cardiovascular Imaging. Eur Heart J Cardiovasc Imaging. 2013 Jul;14(7):61144.

10. Nagueh SF, Smiseth OA, Appleton CP, Byrd BF 3rd, Dokainish H, Edvardsen T, et al. Recommendations for the Evaluation of Left Ventricular Diastolic Function by Echocardiography: An Update from 
th American Society of Echocardiography and the European Association of Cardiovascular Imaging. Eur Heart J Cardiovasc Imaging. 2016 Dec;17(12):1321-1360.

11. Ma W, Zhang W, Shi W, Kong Y, Ma X. Left Ventricular Diastolic Function After Aortic Valve Replacement for Chronic Aortic Regurgitation. Ann Thorac Surg. 2018 Jul;106(1):24-29.

12. Cayli M, Kanadaşi M, Akpinar O, Usal A, Poyrazoğlu H. Diastolic function predicts outcome after aortic valve replacement in patients with chronic severe aortic regurgitation. Clin Cardiol. 2009 Aug;32(8):E19-23.

13. Kim MS, Kim JH, Joo HC, Lee S, Youn YN, Lee SH. Prognostic Markers and Long-Term Outcomes After Aortic Valve Replacement in Patients With Chronic Aortic Regurgitation. J Am Heart Assoc. 2020 Dec 15;9(24):e018292.

14. Morris DA, Belyavskiy E, Aravind-Kumar R, Kropf M, Frydas A, Braunauer K, et al. Potential Usefulness and Clinical Relevance of Adding Left Atrial Strain to Left Atrial Volume Index in the Detection of Left Ventricular Diastolic Dysfunction. JACC Cardiovasc Imaging. 2018 Oct;11(10):14051415.

15. Salas-Pacheco JL, Ávila-Vanzzini N, Eugenia RM, Arias-Godínez JA. Left atrium function by 2D speckle tracking in aortic valve disease. Echocardiography. 2016 Dec;33(12):1828-1834.

16. Rossi A, Tomaino M, Golia G, Santini F, Pentiricci S, Marino P, et al. Usefulness of left atrial size in predicting postoperative symptomatic improvement in patients with aortic stenosis. Am J Cardiol. 2000 Sep 1;86(5):567-70, A9-10.

17. Galli E, Fournet M, Chabanne C, Lelong B, Leguerrier A, Flecher E, et al. Prognostic value of left atrial reservoir function in patients with severe aortic stenosis: a 2D speckle-tracking echocardiographic study. Eur Heart J Cardiovasc Imaging. 2016 May;17(5):533-41.

18. Nolan MT, Thavendiranathan P. Automated Quantification in Echocardiography. JACC Cardiovasc Imaging. 2019 Jun;12(6):1073-1092.

19. Cayli M, Kanadaşi M, Akpinar O, Usal A, Poyrazoğlu H. Relationship between left ventricular geometry and left ventricular systolic and diastolic functions in patients with chronic severe aortic regurgitation. Echocardiography. 2008 Jul;25(6):562-8.

\section{FIGURE LEGENDS.}

Figure 1.

Left atrial (LA) auto-strain analysis. From the apical four chamber view focus in the LA. The software automatically detects and places the LA border and provides the measurement of the average strain for the three major LA function throughout the cardiac cycle: reservoir (LASr), conduit (LAScd) and contractile (LASct) values. The reference point for deformation analysis was at end diastole.

Figure 2.

Receiver operating characteristic (ROC) curves. Area under curve (AUC) was used to evaluate LASr accuracy to predict cardiovascular events $(\mathrm{AUC}=0,78 \mathrm{p}=0,012)$.

Figure 3.

Kaplan-Meier analysis for cardiovascular events. Kaplan-Meier analysis stratified by median value of LASr (less than median of $34 \%$ ) showed that lower LASr values were associated with significantly higher rates of cardiovascular event $(\mathrm{p}=0,013)$.

\section{Table 1.}

Baseline patient characteristics according to the occurrence of the composite outcome during follow-up $(\mathrm{n}=$ $125)$.

Values are expressed as mean \pm standard deviation for continuous variables and No. (\%) for categorical variables. ${ }^{*}$ Adjusted for age and sex. 
CV, cardiovascular; BMI, Body mass index; BNP, B type natriuretic peptide; TR, tricuspid regurgitation; SPAP, Systolic pulmonary artery pressure; LVEDD, left ventricular end diastolic diameter; LVESD, left ventricular end systolic diameter; LVEDV, left ventricular end diastolic volume; LVESV, left ventricular end systolic volume; LVEF, left ventricular ejection fraction; LASct, left atrial strain contractile; LASr, left atrial strain reservoir; LAScd, left atrial strain conduit.

\section{Table 2.}

Cox Regression Analysis between echocardiographic parameters and outcome events.

Univariate analysis: All the echocardiographic systolic and diastolic function parameters assessed.

Multivariate Model 1: Parameters currently used to indicate surgery (LVEDV, LVESV and LVEF) and conventional diastolic variables statistically significant in the univariate model (E, E/e' ratio, LA volume) except SPAP because it is available only in 39 patients.

Model 2: In LA autostrain group. Significant parameters in the Model 1 (LVEDV, E/e' ratio) and LASr.

CI, confidence interval; HR, hazard ratio; LVEDV, left ventricular end-diastolic volume, LVESV, left ventricular end-systolic volume, LVEF, left ventricular ejection fraction; and TR, tricuspid regurgitation.

\section{FIGURES}

Figure 1.

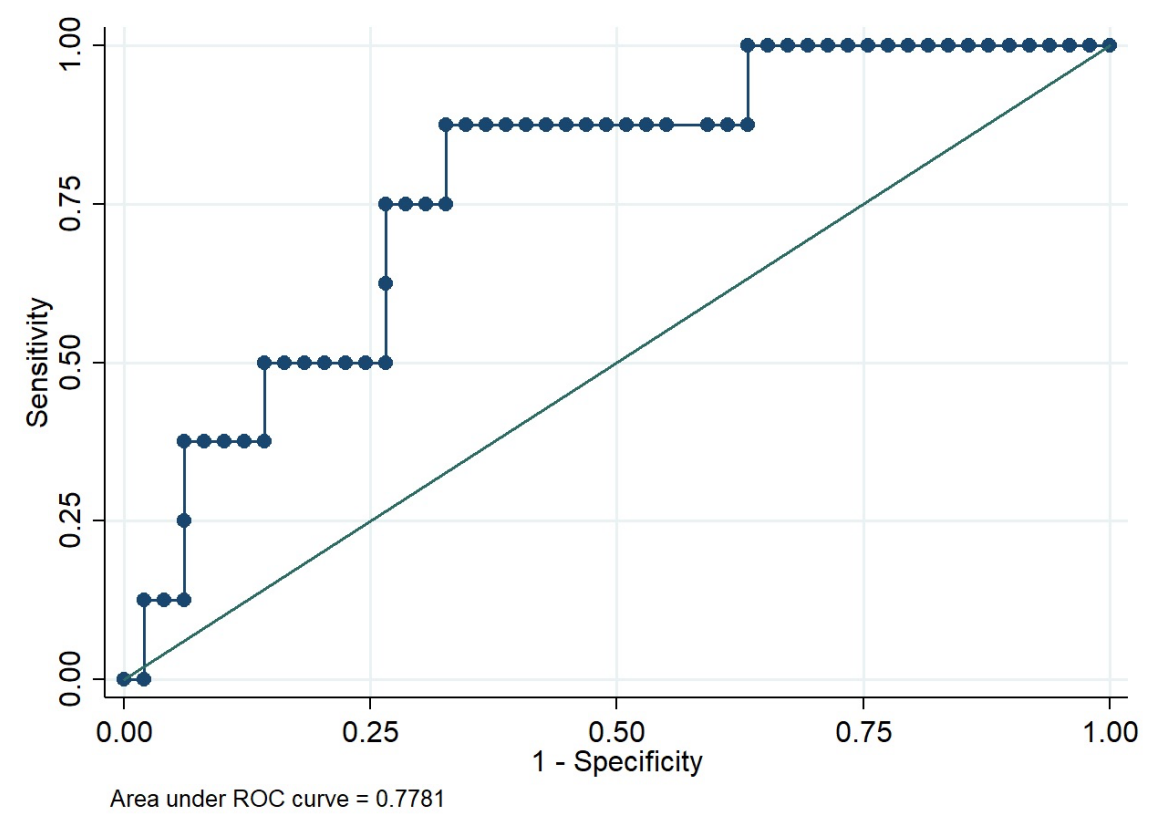

Figure 2 . 


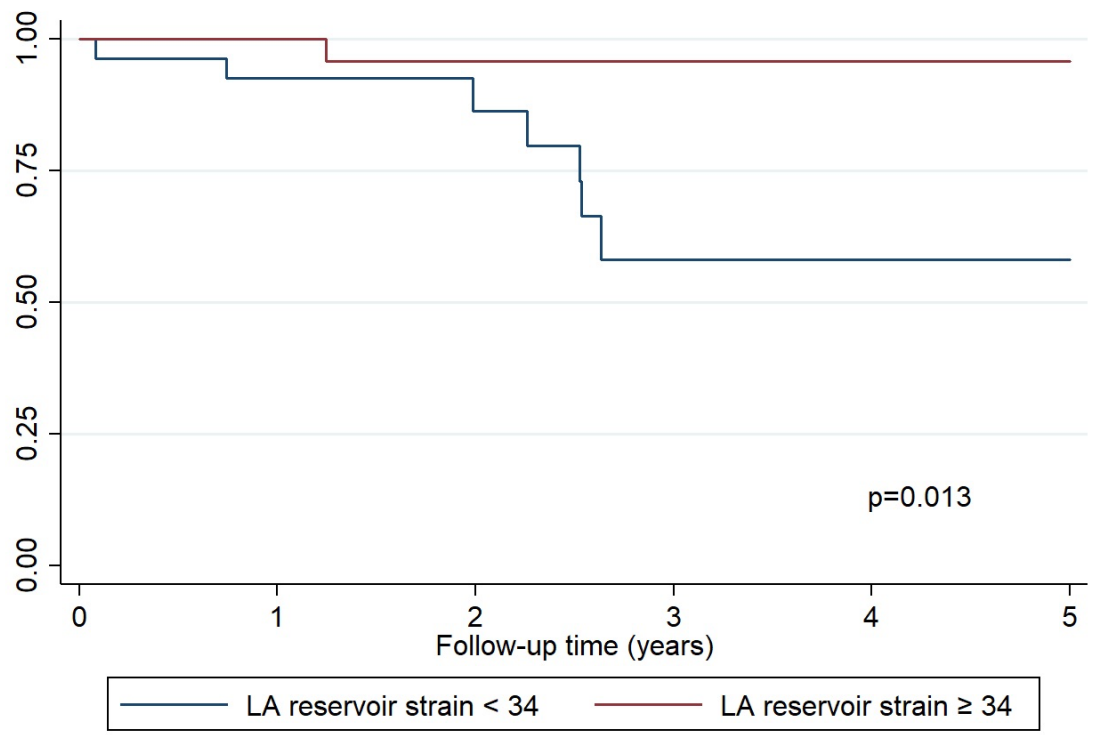

Figure 3.

TABLES.

\begin{tabular}{|c|c|c|c|}
\hline & $\begin{array}{l}\text { Group } 1 \text { (no CV } \\
\text { events) } \mathrm{N}=101\end{array}$ & $\begin{array}{l}\text { Group } 2 \text { (CV events) } \\
\mathrm{N}=25\end{array}$ & $\mathrm{P}^{*}$ \\
\hline $\begin{array}{l}\text { Clinical } \\
\text { characteristics }\end{array}$ & $\begin{array}{l}\text { Clinical } \\
\text { characteristics }\end{array}$ & $\begin{array}{l}\text { Clinical } \\
\text { characteristics }\end{array}$ & $\begin{array}{l}\text { Clinical } \\
\text { characteristics }\end{array}$ \\
\hline Age (years) & $70,8 \pm 17,7$ & $67,0 \pm 15,0$ & 0,40 \\
\hline Female sex $(\%)$ & $44(43,6 \%)$ & $7(28,0 \%)$ & 0,14 \\
\hline BMI & $1,8 \pm 0,2$ & $1,8 \pm 0,2$ & 0,65 \\
\hline Dyslipidemia (\%) & $39(38,6 \%)$ & $6(24,0 \%)$ & 0,31 \\
\hline Diabetes (\%) & $8(7,9 \%)$ & $0(0 \%)$ & 0,98 \\
\hline Hypertension (\%) & $62(61,4 \%)$ & $16(64,0 \%)$ & 0,81 \\
\hline Bicuspid valve (\%) & $19(18,8 \%)$ & $6(24 \%)$ & 0,59 \\
\hline BNP & $197,2(233,5)$ & $83,3(116,7)$ & 0,02 \\
\hline $\begin{array}{l}\text { Echocardiographic } \\
\text { characteristics }\end{array}$ & $\begin{array}{l}\text { Echocardiographic } \\
\text { characteristics }\end{array}$ & $\begin{array}{l}\text { Echocardiographic } \\
\text { characteristics }\end{array}$ & $\begin{array}{l}\text { Echocardiographic } \\
\text { characteristics }\end{array}$ \\
\hline $\begin{array}{l}\text { Vena contracta } \\
(\mathrm{mm})\end{array}$ & $5,7 \pm 1,4$ & $6,3 \pm 1,5$ & 0,84 \\
\hline $\begin{array}{l}\text { Left atrial diameter } \\
(\mathrm{mm})\end{array}$ & $37,0 \pm 6,0$ & $37,0 \pm 7,1$ & 0,99 \\
\hline $\begin{array}{l}\text { Left atrial diameter } \\
\text { (index, } \mathrm{mm} / \mathrm{m} 2 \text { ) }\end{array}$ & $20,8 \pm 3,6$ & $20,0 \pm 3,7$ & 0,94 \\
\hline $\begin{array}{l}\text { Left atrial volume } \\
(\mathrm{ml})\end{array}$ & $53,3 \pm 19,1$ & $66,7 \pm 32,0$ & 0,01 \\
\hline $\begin{array}{l}\text { Left atrial volume } \\
\text { (index. } \mathrm{ml} / \mathrm{m} 2 \text { ) }\end{array}$ & $29,8 \pm 10,1$ & $35,6 \pm 16,1$ & 0,02 \\
\hline $\mathbf{E}$ & $63,5 \pm 22,4$ & $72,0 \pm 26,2$ & 0,03 \\
\hline $\mathbf{A}$ & $79,4 \pm 20,8$ & $86,2 \pm 32,8$ & 0,07 \\
\hline
\end{tabular}




\begin{tabular}{llll}
\hline & $\begin{array}{l}\text { Group 1 (no CV } \\
\text { events) } \mathbf{N = 1 0 1}\end{array}$ & $\begin{array}{l}\text { Group 2 (CV events) } \\
\mathbf{N}=\mathbf{2 5}\end{array}$ & P* \\
\hline E/a & $0,8 \pm 0,4$ & $0,9 \pm 0,5$ & 0,28 \\
E' lat & $7,9 \pm 3,3$ & $7,4 \pm 2,6$ & 0,07 \\
E' med & $6,3 \pm 2,2$ & $6,1 \pm 2,0$ & 0,09 \\
E/e' lat & $9,3 \pm 4,9$ & $10,4 \pm 5,3$ & 0,07 \\
E/e' med & $10,8 \pm 4,5$ & $13,5 \pm 9,2$ & $<0,001$ \\
TR grade & $0,3 \pm 0,5$ & $0,5 \pm 0,6$ & 0,06 \\
SPAP (mmHg) & $29,8 \pm 8,9$ & $35,6 \pm 10,9$ & 0,03 \\
TR (mmHg) & $21,6 \pm 7,6$ & $28,1 \pm 5,8$ & 0,07 \\
LVEDD (mm) & $52,1 \pm 7,0$ & $54,4 \pm 8,7$ & 0,23 \\
LVESD (mm) & $31,8 \pm 5,7$ & $35,3 \pm 8,0$ & 0,06 \\
LVEDDi (mm/m2) & $29,2 \pm 4,2$ & $29,5 \pm 4,6$ & 0,91 \\
LVESDi (mm/m2) & $17,8 \pm 3,6$ & $19,0 \pm 4,5$ & 0,13 \\
LVEDV (ml) & $112,6 \pm 42,9$ & $153 \pm 55,7$ & $<0,01$ \\
LVESV (ml) & $42,4 \pm 24,0$ & $64,8 \pm 37,9$ & $<0,01$ \\
LVEDVi (ml/m2) & $62,0 \pm 19,9$ & $81,9 \pm 25,6$ & $<0,001$ \\
LVESVi (ml/m2) & $23,2 \pm 11,9$ & $34,6 \pm 20,1$ & $<0,01$ \\
LVEF (\%, Teicholz) & $67,5 \pm 9,9$ & $65,9 \pm 10,4$ & 0,13 \\
LVEF (\%, Simpson) & $64,0 \pm 7,7$ & $61,5 \pm 8,4$ & 0,08 \\
LASr & $23,9 \pm 9,0$ & $35,6 \pm 12,5$ & 0,01 \\
LAScd & $-13,3 \pm 4,9$ & $-17,6 \pm 12,3$ & 0,15 \\
LASct & $-11,4 \pm 7,6$ & $-18,9 \pm 6,6$ & 0,01 \\
\hline
\end{tabular}

Table 1.

\begin{tabular}{lllllll}
\hline & HR (95\% CI) & P & HR (95\% CI) & P & HR (95\% CI) & P \\
\hline LVEDV (ml) & $1,01(1,01-1,02)$ & $<0,001$ & $1,02(1,01-1,03)$ & $<0,001$ & $1,05(1,01-1,08)$ & 0,016 \\
LVESV (ml) & $3,85(1,44-10,33)$ & 0,007 & & & - & - \\
LVEF - biplane (\%) & $0,95(0,90-1,00)$ & 0,053 & & - & - \\
E & $1,02(1,00-1,03)$ & 0,015 & & - & - \\
A & $1,01(1,00-1,03)$ & 0,128 & - & - & - \\
E/e' ratio & $1.10(1.04-1.17)$ & 0,002 & $1,12(1,03-1,23)$ & 0,01 & & - \\
TR (grade) & $1,63(0,77-3,44)$ & 0,200 & & & - & - \\
SPAP (mmHg) & $1,10(1,02-1,16)$ & 0,008 & & - & 0,035 \\
LA volume (ml) & $1,02(1,00-1,04)$ & 0,013 & & - & $0,80(0,65-0,98)$ & \\
LASr_ED & $0,93(0,87-0,99)$ & 0,021 & - & & \\
LA diameter (mm) & $1,02(0,94-1,10)$ & 0,637 & & & \\
LVEDD (mm) & $1,03(0,97-1,10)$ & 0,377 & & & & \\
\hline
\end{tabular}

Table 2. 

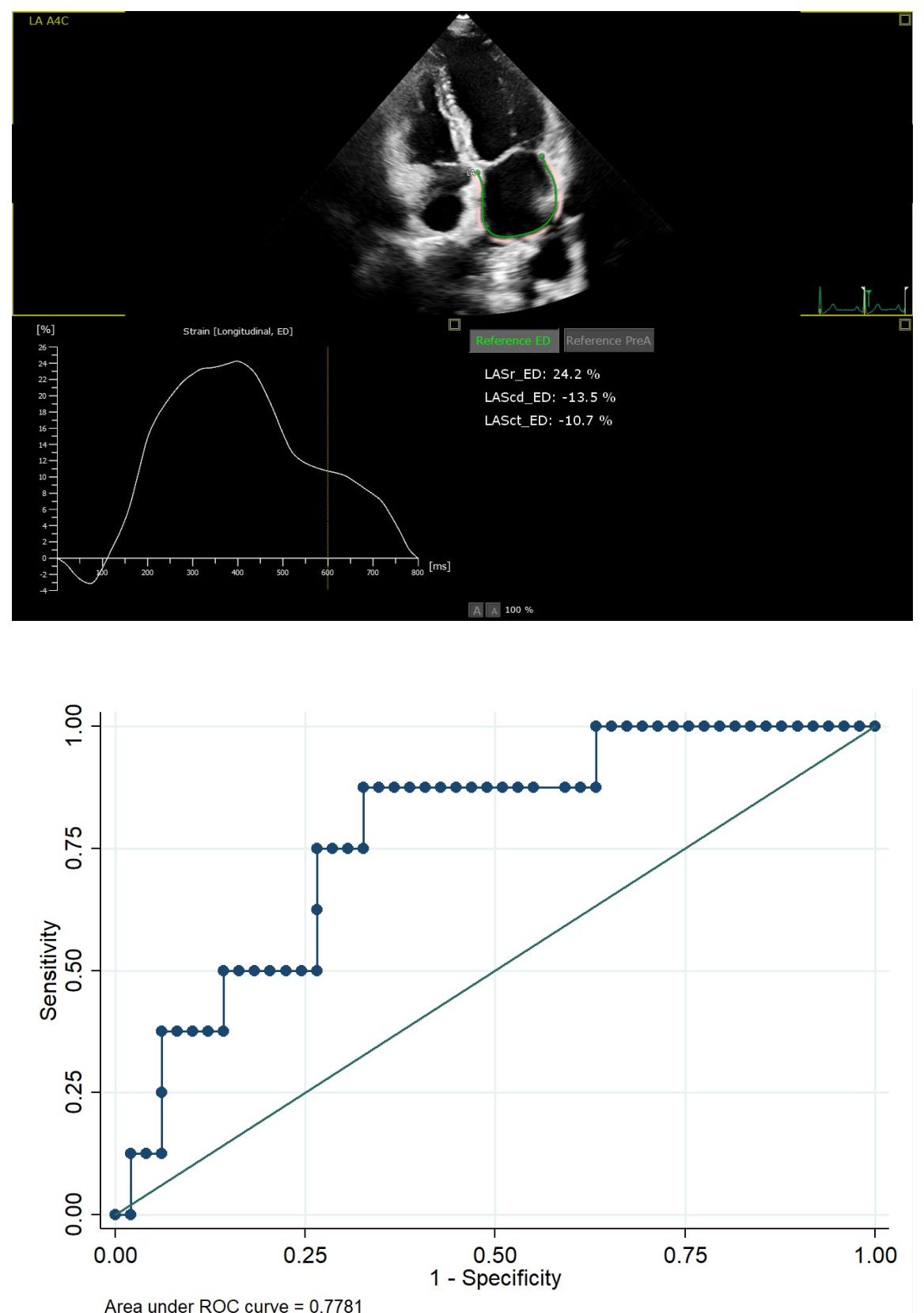


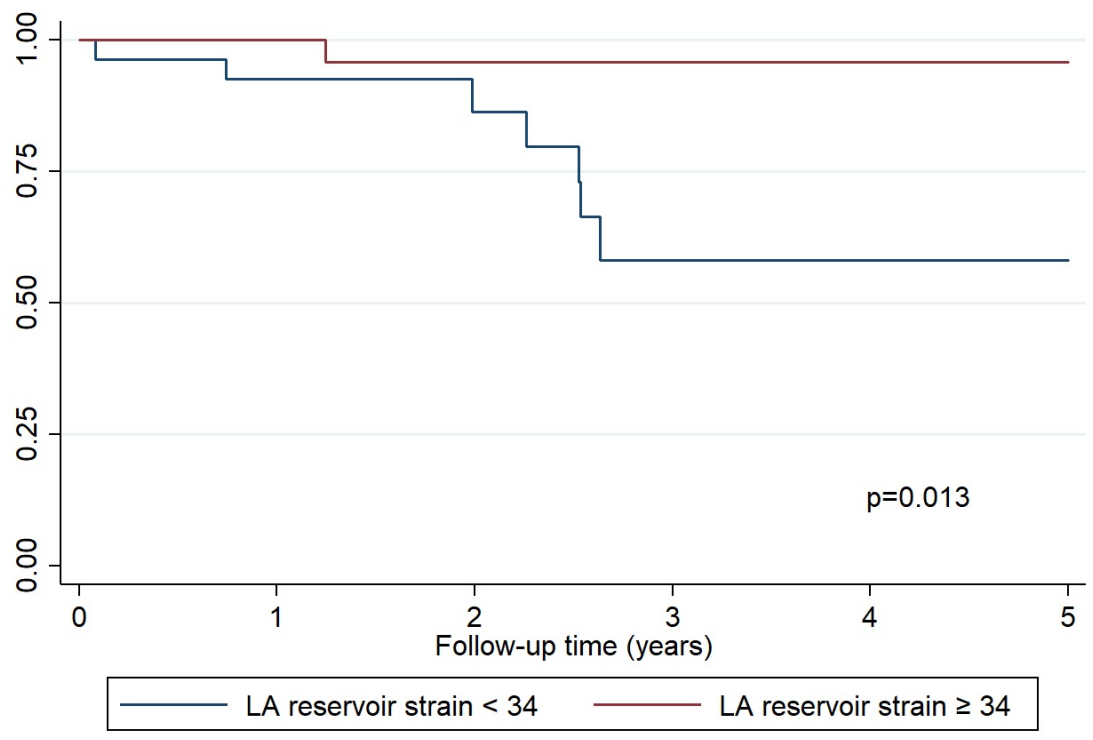

